\title{
ON SINGULAR CHAINS AND CYCLES
}

\author{
BY S. LEFSCHETZ
}

1. Introduction. The theory of the topological invariance of the absolute or relative combinatorial characters of a complex, as developed in our Colloquium Lectures on Topology (Chapter II), was based, following Alexander and Veblen, upon the concept of singular chain. Our presentation, and indeed any known to us, appears to give rise to many misconceptions which it is proposed to clear up in the present note. Unless otherwise stated the notations are those of Topology.

2. Singular Cells. Let $R$ be a topological space and let $e_{p}$ be a simplicial oriented cell such that there exists a continuous singlevalued transformation (=c.s.v.t.) $T$ of the point set $e_{p}$ into a subset $E_{p}$ of $R$, where $E_{p}=T e_{p}$. The symbol $\left(e_{p}, T, E_{p}\right)$, associated with the set $\bar{E}_{p}$ is called a singular oriented p-cell on $R$. If $e_{p}^{\prime}$ is another $e_{p}$, there exists a barycentric transformation $U$ of $\bar{e}_{p}^{\prime}$ into $\bar{e}_{p}: U \bar{e}_{p}^{\prime}=\bar{e}_{p}$. If we set $T^{\prime}=T U$, it is evident that $\left(e_{p}^{\prime}, T^{\prime}, E_{p}\right)$ defines also a singular oriented $p$-cell on $R$. We shall agree to consider it as identical with the first:

$$
\left(e_{p}^{\prime}, T^{\prime}, E_{p}\right)=\left(e_{p}, T, E_{p}\right) .
$$

This has the advantage of freeing the notion of singular cell from a too narrow connection with a specific image $e_{p}$.

3. Singular Chains. The singular $p$-chain $C_{p}$ on $R$ is now defined as the association of a symbol

$$
C_{p}=\sum t_{i}\left(e_{p}^{i}, T^{i}, E_{p}^{i}\right)
$$

with coefficients $t$ belonging to one of the three rings (rational numbers, integers, integers $\bmod m$ ) considered in Topology, together with the set of all sets $\bar{E}_{p}^{i}$ corresponding to $t^{\prime} \mathrm{s} \neq 0$. As a special case the $e$ 's might be cells of a finite complex $k$ such that there exists a c.s.v.t. $T$ of $k$ into a subset of $R$. Then the chain symbol may take the form

$$
C_{p}=\sum t_{i}\left(e_{p}^{i}, T, E_{p}^{i}\right),
$$

and $C_{p}$ may be considered as the image of the subchain 


$$
c_{p}=\sum t_{i} e_{p}^{i}
$$

of $k$, but that is not essential. In this instance we might have represented $C_{p}$ by the symbol $\left(\sum t_{i} e_{p}^{i}, T, \sum t_{i} E_{p}^{i}\right)$ analogous to the cell symbol. Observe also that we may find for any chain (2) an equivalent representation (3). For we may take the cells $e_{p}^{i}$ to be simplexes in some $S_{n}$ whose closures do not meet, then define $T$ as coincident with $T^{i}$ on $e_{p}^{i}$. The closure of the sum of the cells $e_{p}^{i}$ will then be $k$, and (2) will assume the form (3).

If we have several singular chains $C_{p}{ }^{i}$, then $\sum s_{i} C_{p}{ }^{i}$, where the coefficients $s_{i}$ belong to the same ring as those of the chains, defines a $p$-chain which is called the linear combination with coefficients $s_{i}$ of the chains $C_{p}^{i}$. We have thus moduli of singular $p$-chains wholly analogous to the moduli of subchains of a complex.

4. Boundary Relations. Returning to $\left(e_{p}, T, E_{p}\right)$, let the boundary relations for $e_{p}$ be

$$
e_{p} \rightarrow \sum \eta_{i} e_{p-1}^{i}=F\left(e_{p}\right) .
$$

Since $T$ is a transformation of $\bar{e}_{p}$ into $\bar{E}_{p}$, it transforms $e_{p-1}^{i}$ into a subset $E_{p-1}^{i}$ of $\bar{E}_{p}$ and hence $\left(e_{p-1}^{i}, T, E_{p-1}^{i}\right)$ is a singular $(p-1)$-cell on $R$. The singular $(p-1)$-chain

$$
F\left(e_{p}, T, E_{p}\right)=\sum \eta_{i}\left(e_{p}^{i}, T, E_{p-1}^{i}\right)
$$

is called the boundary of $\left(e_{p}, T, E_{p}\right)$ and we write here also

$$
\left(e_{p}, T, E_{p}\right) \rightarrow F\left(e_{p}, T, E_{p}\right) .
$$

The boundary of the chain (2) is now by definition

$$
F\left(C_{p}\right)=\sum t_{i} F\left(e_{p}^{i}, T^{i}, E_{p}^{i}\right),
$$

for which we also write

$$
C_{p} \rightarrow F\left(C_{p}\right) .
$$

Owing to (1) this boundary depends solely on $C_{p}$ but not on the particular transformations $T^{i}$ that occur in (8). Let $C_{p}$ be in the special form (3) with an associated non-singular image (4), and let the boundary relation for $c_{p}$ be

$$
c_{p} \rightarrow \sum s_{i} e_{p-1}^{i} .
$$


Then we have well defined singular cells $\left(e_{p-1}^{i}, T, E_{p-1}^{i}\right)$ and we find that

$$
C_{p} \rightarrow \sum s_{i}\left(e_{p-1}^{i}, T, E_{p-1}^{i}\right),
$$

which may be described by the statement: the boundary of a singular image of a chain is the singular image of the boundary of the chain.

5. Degenerate Case. Let $R$ undergo a c.s.v.t. $U$ into a new space $R^{\prime}$. Then the singular cell $\left(e_{p}, T, E_{p}\right)$ will go over into a singular $p$-cell $\left(e_{p}, U T, U E_{p}\right)$ and $C_{p}$ into

$$
U C_{p}=\sum t_{i}\left(e_{p}^{i}, U T^{i}, U E_{p}^{i}\right) .
$$

To different representations of the same singular $p$-cell on $R$ there will merely correspond different representations of the same singular $p$-cell on $R^{\prime}$, and to $F\left(C_{p}\right)$ there will now correspond $F\left(U C_{p}\right)$. In particular also if $C_{p} \equiv 0$, likewise $U C_{p} \equiv 0$.

The preceding observations have an immediate application to degenerate cells. Let $\left(e_{p}, T, E_{p}\right)$ be a singular cell on $R$, and let us suppose that there exists a simplex $\sigma_{q}, q<p$, and two c.s.v.t.'s $T^{\prime}, T^{\prime \prime}$, such that $T^{\prime}$ is a simplicial transformation of $e_{p}$ into $\sigma_{q}$ and that $T^{\prime \prime} \cdot T^{\prime}=T$. The cell $\left(e_{p}, T, E_{p}\right)$ is called a singular degenerate $p$-cell on $R$ and chains made up exclusively of such cells are called degenerate chains. If $R^{\prime}=U R$ as above, the degenerate cells and chains of $R$ go over into degenerate cells and chains of $R^{\prime}$.

According to Topology, Chapter II, No. 2, $F\left(e_{p}, T^{\prime}, \sigma_{q}\right)$ is a degenerate $(p-1)$-chain, and hence when $\left(e_{p}, T, E_{p}\right)$ is degenerate so is its boundary. Hence this holds likewise as regards degenerate $p$-chains. Let us agree to consider all degenerate chains as identically zero. By the observation just made degenerate chains will then completely disappear from all boundary relations.

6. Homologies. From the preceding section it appears clearly that when $R$ and $R^{\prime}$ are homeomorphic, the homeomorphism between them associates respectively to one another their moduli of $p$-chains, of bounding $p$-chains and their degenerate $p$-chains. These are therefore topological and the homology characters derived from the moduli are topological invariants.

Regarding these homologies, we introduce them exactly as for complexes. In particular if $A$ intersects $B$ in a set $A \cdot B$ closed 
relatively to $B$, the neglect of the singular cells $\left(e_{p}, T, E_{p}\right)$, such that $E_{p} \subset A$, leads to the characters of $B \bmod A$.

7. Invariance of the Combinatorial Homology Characters. Suppose now that $R$ itself is a finite simplicial complex $K$ and let $\boldsymbol{\epsilon}_{p}^{i}$ designate its cells. They can be considered as the singular cells $\left(\epsilon_{p}^{i}, 1, \epsilon_{p}^{i}\right)$ and it is readily seen that the formal singular boundary relations involving these cells alone are the same as the combinatorial relations between the cells $\epsilon_{p}^{i}$ themselves. Therefore whenever only singular cells of this type are involved, the singular boundary relations (7) for $K$ are reduced to the combinatorial relations.

The invariance of the combinatorial homology characters of $K$ is established by identifying them with the corresponding topological characters. The steps in the proof are as follows.

(a) Let $C_{p}$ be a singular chain on $K$ which we assume henceforth in the simplified form (11) with $T$ and the non-singular prototype $c_{p}$ fixed. There exists an $\eta>0$ depending on $K$ but not on $C_{p}$, such that when mesh $C_{p}<\eta$, the chain can be homotopically deformed into a subchain $C_{p}{ }^{\prime}$ of $K$, the deformation keeping each cell on the closure of the cell of $K$ that carries it. This is the deformation theorem (Topology, p. 86). It implies (loc. cit., p. 78) that there are deformation chains, all singular, indicated by $\mathcal{D}$, such that

$$
\begin{gathered}
\mathcal{D} C_{p} \rightarrow C_{p}^{\prime}-C_{p}-\mathcal{D} F\left(C_{p}\right), \\
C_{p}^{\prime}=\sum s_{i} E_{p}^{i} .
\end{gathered}
$$

(b) If mesh $C_{p}>\eta$ the chain has a subdivision chain $C_{p}$ whose mesh is suitable. Subdivision is defined as in Topology (p. 85), by reference to a subdivision of $c_{p}$.

(c) Suppose that $C_{p}$ possesses certain cells (not necessarily $p$-cells) which belong to $K$ and whose sum is therefore a subcomplex $K_{q}$ of $K$. Then the subdivision and deformation in (b) may be so chosen as to leave $K_{q}$ fixed point for point. The proof indicated in Topology (p. 87, Remark I) only shows that $C_{p}$ may be so modified as to leave the cells of $K_{q}$ invariant individually but not point for point. The more accurate result, which is of interest for its own sake, is proved as follows. We show by induction as in Topology (p. 86) that the deformations there indicated leave $K_{q}$ invariant point for point provided that 
any cell of $C_{p}$ without vertices on $K_{q}$ is of diameter $<\eta$, and that a cell having a face in common with $K_{q}$ has all its points not farther than $\eta$ from that face. If $C_{p}$ does not fulfill these conditions we find by reference to $c_{p}$ that a suitable subdivision of $C_{p}$ without new vertices on $K_{q}$ will behave as required. For if $c_{p}$ is a subchain, say of $k$, there is a subcomplex $k^{\prime}$ of $k$ such that $T \cdot k^{\prime}=K_{q}$. We can then apply to $k$ a series of subdivisions differing from regular subdivisions only in so far that no new vertices are ever introduced on $k^{\prime}$. Given any $\zeta$ we can thus obtain a subdivision $c_{p}^{*}$ of $c_{p}$ whose cells fulfill relatively to $k^{\prime}$ and $\zeta$ the two conditions that we wish to impose upon the cells of $C_{p}$ relatively to $K_{q}$ and $\eta$. Since $T$ is continuous the required result follows for $C_{p}$.

Consider now the boundary relations $\bmod L$, where $L$ is a subcomplex of $K$. Let $\Gamma_{p}$ be a (singular) cycle $\bmod L$. By (a) there is a subdivision $\Gamma_{p}{ }^{\prime}$ of $\Gamma_{p}$ homotopically deformable into a subcycle $\Gamma_{p}^{*}$ of $K$, its points on $L$ remaining on $L$, and $\Gamma_{p}^{\prime} \sim \Gamma_{p} \bmod$ $L$ on $\Gamma_{p}$ itself (Topology, p. 87) and hence a fortiori on $K$. By (c), if $C_{p+1} \rightarrow \Gamma_{p}^{\prime} \bmod L$, there is a subdivision $C_{p+1}{ }^{\prime}$ of $C_{p+1}$ with the same boundary $\Gamma_{p}{ }^{\prime}$, deformable into a subchain $C_{p+1}{ }^{*}$ by a homotopy leaving $\Gamma_{p}^{\prime}$ invariant, so that $C_{p+1}^{*} \rightarrow \Gamma_{p}^{\prime} \bmod L$. Therefore if the initial cycle $\sim 0 \bmod L$ in the topological sense, the reduced cycle $\sim 0 \bmod L$ in the combinatorial sense. From this follows immediately as in Topology (p. 88), that the topological and combinatorial homology groups of the same types are simply isomorphic and hence have the same numerical invariants. Therefore the combinatorial homology characters are topological invariants.

8. Remarks. I. Once the notion of singular cell has become familiar one will naturally abandon the explicit (too explicit) $(e, T, E)$ notation in favor of the simpler $E$ of Topology.

II. The following circumstance may arise in connection with our definition of singular cell. Taking for the sake of simplicity $p=2$, let $e_{2}=A B C$ be an (oriented) isosceles triangle with $A B$ $=A C$ and let $A D$ be the altitude issued from $A$. Let $U$ be the symmetry about $A D$ and $T$ a transformation $=1$ on $A D B$, $=U$ on $A D C$. If we set $T^{\prime}=T \cdot U, e_{2}^{\prime}=A C B$, we have

$$
\left(e_{2}, T, E_{2}\right)=\left(e_{2}^{\prime}, T U, E_{2}\right)=\left(-e_{2}, T U, E_{2}\right)=\left(-e_{2}, T, E_{2}\right)
$$


and therefore

$$
2\left(e_{2}, T, E_{2}\right)=0 \text {. }
$$

Owing to this, E. Čech, who pointed out this circumstance to us, suggested that in the present and in the similar instance for any $p$, the singular cell be also considered as degenerate. The more extended meaning to be thus attached to degenerate cells, while justifiable, is not however essential.

Princeton University

\section{VARIABLES CORRELATED IN SEQUENCE*}

BY A. T. CRAIG

1. Introduction. If each of $n$ variables, $x_{1}, x_{2}, \cdots, x_{n}$, represents a quantitative character of an individual, and if the variables are correlated in sequence, that is, $x_{1}$ is correlated with $x_{2}, x_{2}$ is correlated with $x_{3}, \cdots$, and in general $x_{i}$ is correlated with $x_{i+1}$, it seems natural to inquire about the correlation between a character, say $x_{1}$, of one individual and a character, say $x_{3}$, of a second individual, with the condition imposed that the two individuals have identical measurements with regard to the character $x_{2}$. It is this problem with which we shall be primarily concerned in the present paper. As we proceed, we shall place appropriate restrictions upon the nature of the correlation which exists between the variables. We shall, however, make no assumptions regarding the correlation between the variables other than that between them in adjacent pairs.

In order to provide a convenient point of departure and to exhibit a set of variables correlated in sequence, we shall first consider a rather elementary problem which arises when measurements are made under a constant law of probability.

2. The Correlation between Measurements under a Constant Law of Probability. Let the variable $t$ obey a constant law of probability $f(t)=1 / a, 0 \leqq t \leqq a$. Let successive sets of $n$ independent measurements each, say $t_{1}, t_{2}, \cdots, t_{n}$, be made upon $t$. We may, without loss of generality, suppose the meas-

* Presented to the Society, April 8, 1932, under the title Some properties of correlated variables. 